\title{
Minimally-invasive 2-Stage Liver Resection with Portal Vein Ligation for Bi-Lobar Colorectal Liver Metastases
}

\author{
Nan-Zun Teo ${ }^{1}$, Dawn Chong ${ }^{2}$, Brian K. P. Goh ${ }^{1,3}$
}

Corresponding author:

Dr Brian K.P. Goh

Senior Consultant and Professor

Singapore General Hospital, Level 5,

20 College Road, Academia,

Singapore 169856

E-mail: bsgkp@hotmail.com
'Department of Hepatopancreatobiliary and Transplant Surgery, Singapore General Hospital ${ }^{2}$ Department of Medical Oncology, National Cancer Center Singapore

${ }^{3}$ Duke-NUS Medical School, Singapore

\section{ABSTRACT}

Metastectomy is the only potentially curative treatment option for patients with colorectal liver metastases. It has been shown to improve long term survival in this group of patients. However, patients with extensive colorectal liver metastases with borderline resectability often pose a therapeutic challenge to the surgeon. There is a potential risk of liver failure from insufficient liver remnant following metastectomy. In this instance, a two stage liver resection has emerged as a viable option. We describe our technique for a completely minimally invasive approach to two stage liver resection for a patient with bi-lobar colorectal liver metastases. The patient remains disease free and well after 1 year and 2 months.

Key words: colon cancer, minimally invasive surgery, neoplasm metastasis

\section{INTRODUCTION}

Laparoscopic liver resection (LLR) was first described by Gagner et al in 1992 (1). It was first performed for benign liver tumors but its use has now been expanded to include resection for malignant tumors and even major hepatectomies $(2,3)$. In comparison to open hepatectomies, its benefits include a shorter length of stay, decrease intra-operative blood loss and reduced postoperative complications $(4,5)$. The techniques for LLR continue to evolve $(6-8)$ and in recent years, robotic assisted laparoscopic surgery has also been added to the armamentarium of the liver surgeon (9). We describe a challenging case of descending colon cancer with multiple bi-lobar liver metastasis who successfully underwent 2-stage robotic assisted laparoscopic liver resection with intraoperative portal vein ligation.

\section{DESCRIPTION OF SURGICAL TECHNIQUE}

\section{Patient selection}


this technically challenging procedure. The patient should have liver-confined metastasis and due consideration should be made to ensure adequate future liver remnant following oncological resection of the liver metastases. The patient should also be thoroughly evaluated pre-operatively to ensure fitness for operation, and ability to tolerate pneumoperitoneum. It is important to emphasize that these cases should always be discussed in a multidisciplinary tumor board. The patient is a 61-year-old female with descending colon cancer with synchronous bi-lobar liver metastases. The liver metastases were in segments 2, 3, 5 and 8 (figs. 1 - 5). After discussion at the multidisciplinary tumor board, the decision was made for her to undergo colon resection followed by adjuvant chemotherapy. Liver resection of the bilobar liver metastases was to be considered there after. The patient first underwent a laparoscopic left hemicolectomy. This was followed by a course of chemotherapy for 2 months with 3 cycles of combined Capecitabine and Oxaliplatin. She was then restaged and confirmed to have liver limited metastases which had decreased in size. She was subsequently planned for a 2-stage liver resection as her future liver remnant after right hepatectomy and segment $2 \beta$ metastectomy was calculated to be less than $25 \%$. The $1^{\text {st }}$ stage would include resection of all lesions in segments $2 \beta$ of the liver and right portal vein ligation. Subsequently, the $2^{\text {nd }}$ stage right hepatectomy would then be performed if there was adequate hypertrophy of the left liver lobe.

\section{SURGICAL TECHNIQUE}

The $1^{\text {st }}$ stage of the operation was performed with the Da Vinci Si robot. The patient was put in the supine position. A $12 \mathrm{~mm}$ supra-umbilical incision was made for the camera port. Two $8 \mathrm{~mm}$ robot ports were placed in the right hypochondrium and another $8 \mathrm{~mm}$ robotic port was placed in the left hypochondrium. A $12 \mathrm{~mm}$ assistant port was placed in the right iliac fossa. The robot was docked at the 12-0 clock position. Adhesiolysis was performed and an intra-operative ultrasound (IOUS) done to confirm the location of the lesions. A total of 4 lesions were identified in the left lobe, two in segment 2, one in segment 3 and another spanning segments 2 and 3 . These were resected via metastectomy and left lateral subsectionectomy. The hilum was looped in preparation for Pringle's maneuver. Routine cholecystectomy was performed. Resection of the liver metastases with adequate margins was performed using ultrasonic dissection and the powered endoscopic stapler. The right hepatic

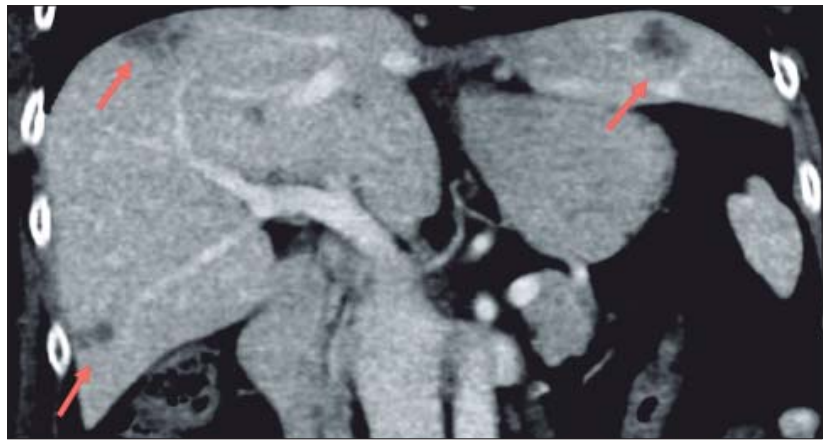

Figure 1 - Coronal CT showing multiple liver metastases in segments 2, 3, 5 and 8

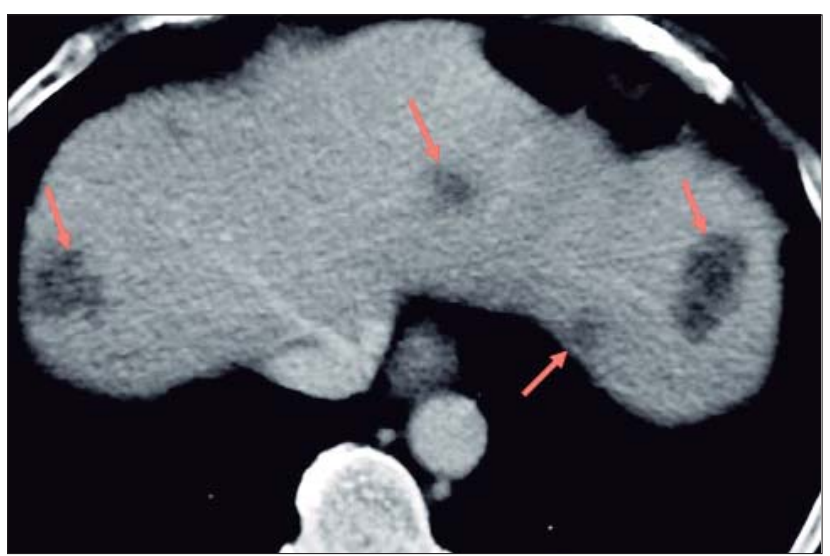

Figure 2 - Axial CT showing multiple liver metastases in segments 2, 3, 5 and 8

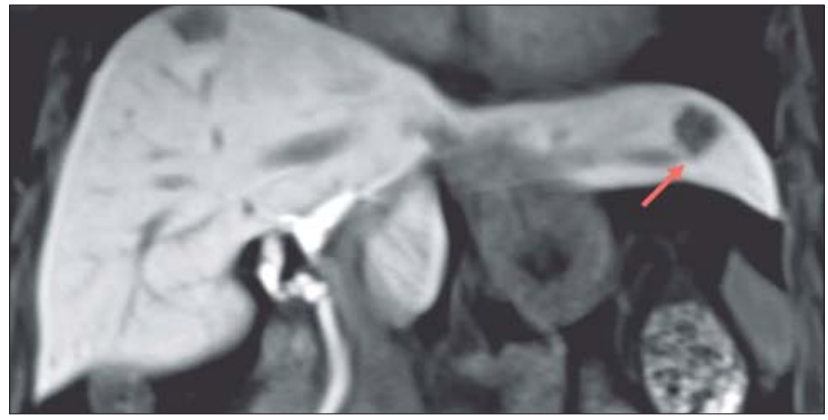

Figure 3 - Coronal MRI liver showing showing multiple liver metastases in segments 2,3

hilum was then dissected and the right portal vein identified. The right portal vein was ligated with a silk $2 / 0$ tie and a ligation clip. The right hepatic artery was isolated and slung with a prolene tie. The specimens were then retrieved using a large endobag through a 3 $\mathrm{cm}$ midline incision.

A computed tomography scan of the liver with volumetry calculation was performed 3 weeks after the $1^{\text {st }}$ stage which showed left lobe hypertrophy. The future liver remnant after right hepatectomy was now 


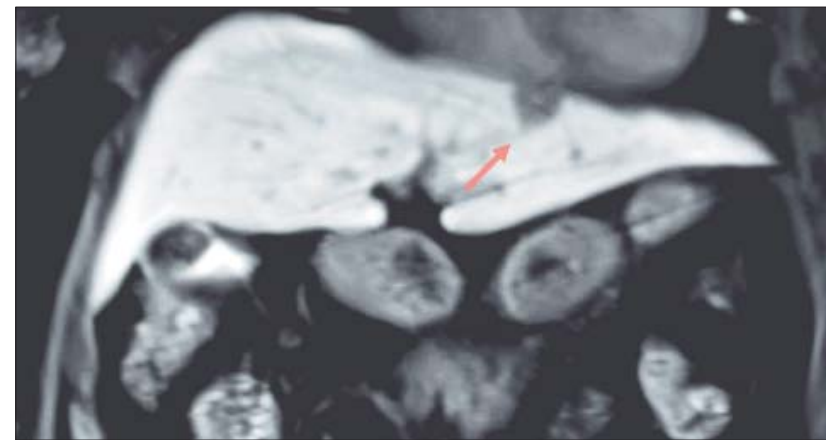

Figure 4 - Coronal MRI liver showing showing multiple liver metastases in segments 2, 3

$550 \mathrm{mls}$ (45.1\%) (fig. 6). The patient subsequently underwent 2nd stage totally laparoscopic right hepatectomy 4 weeks after the 1st stage liver resection.

She was placed in the supine position. A supraumbilical incision was made and the abdomen entered. A $12 \mathrm{~mm}$ right paraumbilical, one $5 \mathrm{~mm}$ right hypochondrial, one $12 \mathrm{~mm}$ right hypochondrial, one 5 $\mathrm{mm}$ epigastric, one $5 \mathrm{~mm}$ left hypochondriac and one $12 \mathrm{~mm}$ right iliac fossa port was placed under direct vision. Adhesiolyis was then performed. The right coronary ligament, right triangular ligament and the falciform ligament was dissected using a combination of the ultrasonic shears and hook diathermy to mobilize the liver. IOUS was then performed to identify and confirm the right lobe metastases. Dense adhesions were noted at the extrahepatic right pedicle due to the previous portal vein ligation. IOUS was used to identify the right glissonian pedicle including the right anterior and posterior pedicles. These were approached via the intrahepatic glissonian approach whereby the liver parenchyma was transected to expose the right glissonian pedicles. The right anterior and posterior glissonian pedicles were exposed and stapled separately using apowered endoscopic stapler. The resection line was than marked along the ischemic line. Parenchymal transection with the ultrasonic shears was performed along Cantlie's line. The right hepatic vein was identified intrahepatically and transected with a powered endoscopic stapler. The cut surface of the liver was meticulously inspected for hemostasis and fibrin glue applied. The right hepatectomy specimen was retrieved with a large endobag via an $7 \mathrm{~cm}$ midline wound and routine closure performed.

\section{Outcome}

The $1^{\text {st }}$ stage operation took 355 minutes, with minimal blood loss and no blood transfusions were

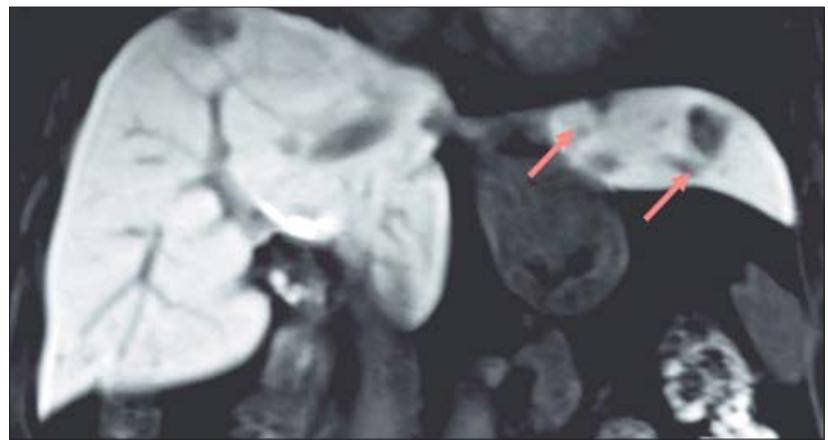

Figure 5 - Coronal MRI liver showing showing multiple liver metastases in segments 2,3

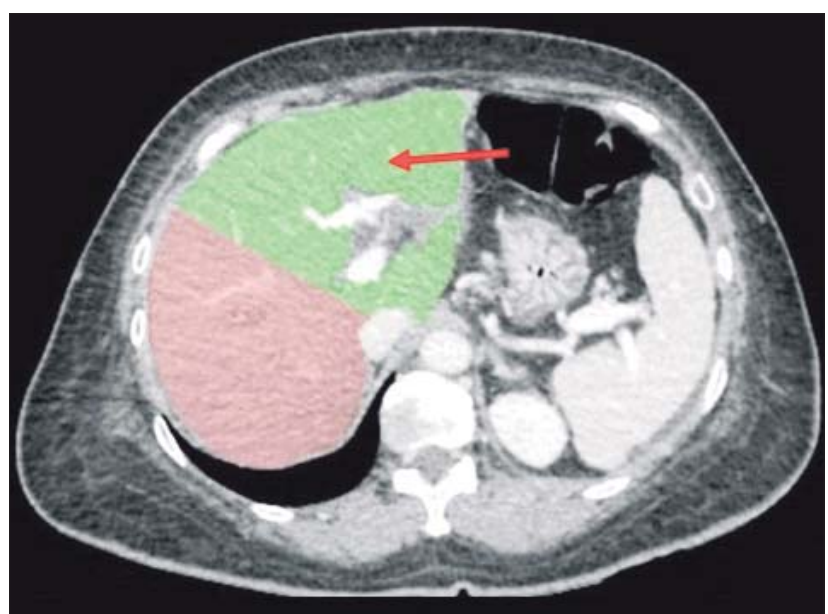

Figure 6 - CT volumetry showing future liver remnant following right hemihepatectomy

required. The $2^{\text {nd }}$ stage operation took 345 minutes and likewise the patient was hemodynamically stable throughout with minimal blood loss and did not require any blood transfusion. The patient recovered well from both surgeries and was discharged on the $4^{\text {th }}$ and $9^{\text {th }}$ post-operative day respectively. Histopathological assessment of the specimen revealed findings consistent with metastatic colon adenocarcinoma with clear resection margins. The patient has currently completed her planned 8 cycles of combined Capecitabine and Oxaliplatin adjuvant therapy and is recurrence free 6 months postoperatively.

\section{DISCUSSION}

Many studies report favorable outcomes after open two stage hepatectomies (TSH) for bi-lobar colorectal liver metastases $(10,11)$ and more recently some authors have described a MIS approach to TSH $(12,13)$. Fuks et al reported acceptable short-term outcomes such as a low conversion rate, minimal blood loss and 
low morbidity rates in their series of 26 patients who underwent a pure laparoscopic approach for TSH (12). Furthermore, they also reported comparable oncological outcomes to the open approach with 78 and 41 percent overall survival at three and five years respectively (12). Kilburn et al describe a laparoscopic 1st stage and open 2nd stage approach to patients with bi-lobar colorectal liver metastases. The authors describe a benefit of fewer adhesions at the $2^{\text {nd }}$ stage operation with this approach.Furthermore, they added that laparoscopic portal vein ligation during the first stage was feasible and can save the patient a separate portal embolization procedure (13).

The MIS approach to the second stage is however more technically challenging as there are anatomical alterations in the hypertrophied remnant liver after portal vein ligation and chemotherapy induced liver injury (12). Furthermore, dense adhesions can be encountered at the hilum. In this patient, we elected to performan intra-hepatic glissonian approach to the right portal pedicle to avoid injury to critical hilar structures. Due to the technical difficulty of these cases, we recommend that these cases should only be attempted by surgeons with considerable experience with LLR. Prior experience with laparoscopic repeat liver resections for recurrent tumors would also be useful as surgeons would be experienced with dealing with potentially dense adhesions around the liver and hilum $(14,15)$. Our institution had performed more than 300 cases of LLR including more than 20 laparoscopic repeat liver resections and before attempting this procedure. Also, the surgeon performing this procedure has performed more than 500 partial liver resections and over 150 laparoscopic liver resections since completing surgical training and prior to attempting this procedure.

\section{CONCLUSION}

In conclusion, a totally MIS strategy for two stage liver resection of bi-lobar liver metastases is feasible in a carefully selected patient. The addition of the Da Vinci robot to the armementarium of the surgeon may allow for easier hilar dissection and portal vein ligation.

\section{Disclosure}

This study did not receive any funding or financial support.

\section{REFERENCES}

1. Gagner M, Rheault M, Dubuc J. Laparoscopic partial hepatectomy for liver tumor (abst). Surg Endosc. 1992;6:99.

2. Dagher I, O'Rourke N, Geller DA, Cherqui D, Belli G, Gamblin TC, et al. Laparoscopic major hepatectomy: an evolution in standard of care. Ann Surg. 2009;250:856-860.

3. Goh BK, Teo JY, Chan CY, Lee SY, Cheow PC, Chow PK, et al. Evolution of laparoscopic liver resection at Singapore General Hospital: a nine-year experience of 195 consecutive resections. Singapore Med J 2017;58(12):708-713.

4. Mirnezami R, Mirnezami AH, Chandrakumaran K, Abu Hilal M, Pearce NW, Primrose JN, et al. Short- and long-term outcomes after laparoscopic and open hepatic resection: systematic review and meta-analysis. HPB (0xford). 2011;13:295-308.

5. BK Goh, CY Chan, JS Wong, SY Lee, VT Lee, PC Cheow, et al. Factors associated with and outcomes of open conversion after laparoscopic minor hepatectomy: Initial experience at a single institution. Surg Endosc. 2015;29:2636-2642.

6. Agcaoglu 0, Aliyev S, Karabulut K, El-Gazzaz G, Aucejo F, Pelley R, et al. Complementary use of resection and radiofrequency ablation for the treatment of colorectal liver metastases: an analysis of 395 patients. World J Surg. 2013;37:1333-1339.

7. Buell JF, Gayet B, Han HS, Wakabayashi G, Kim KH, Belli G, et al. Evaluation of stapler hepatectomy during a laparoscopic liver resection. HPB (Oxford). 2013;15:845-850.

8. Hasegawa Y, Koffron AJ, Buell JF, Wakabayashi G. Approaches to laparoscopic liver resection: a meta-analysis of the role of handassisted laparoscopic surgery and the hybrid technique. J Hepatobiliary Pancreat Sci. 2015;22:335-341.

9. Ho C, Wakabayashi G, Nitta H, Ito N, Hasegawa Y, Takahara T. Systematic Review of Robotic Liver Resection. Surg Endosc. 2013; 27:732.

10. Adam R, Laurent A, Azoulay D, Castaing D, Bismuth H. Two-stage hepatectomy: a planned strategy to treat irresectable liver tumors. Ann Surg. 2000;232: 777-785.

11. Wicherts DA, Miller R, de Haas RJ, Bitsakou G, Vibert E, Veilhan LA et al. Long-term results of two-stage hepatectomy for irresectable colorectal cancer liver metastases. Ann Surg. 2008;248:994-1005.

12. Fuks D, Nomi T, Ogiso S, Gelli M, Velayutham V, Conrad C et al. Laparoscopic two-stage hepatectomy for bilobar colorectal liver metastases. Br J Surg. 2015;102(13):1684-1690.

13. Kilburn DJ, Chiow KH, Lewin J et al. Laparoscopic approach to a planned two-stage hepatectomy for bilobar colorectal liver metastases. ANZ J Surg. 2016;86:811-815.

14. Goh BK, Teo JY, Chan CY et al. Laparoscopic repeat liver resection for recurrent hepatocellular carcinoma. ANZ J Surg. 2017; 87(10):E143-E146.

15. Goh BK, Teo JY, Chan CY, et al. Review of 103 cases of laparoscopic repeat liver resection for recurrent hepatocellular carcinoma. J Laparoendosc Adv Surg Tech A. 2016; 26(11):876-881. 